$\mathrm{XX}$.

Aus der 1. und IlI. med. Abt. (Vorstände: Prim.-Prof. Dr. G. Singer und Prim.-Doc. Dr. H. Weinberger) und dem path.-anat. Inst. (Vorstand: Hofrat Prof. Dr. R. Paltauf) der k. k. Krankenanstalt „Rudolfstiftung ${ }^{k}$ zu Wien.

\title{
Beschleunigter Nachweis der Tuberculose im Tierversuch durch Milzimpfung.
}

Von

\author{
Dr. M. Damask und Dr. F. Schweinburg, \\ Assistenten der $\mathbf{A b t e l l u n g e n .}$
}

Dank den verschiedenen Anreicherungsverfahren, insbesondere mit Antiformin, ist es heute leichter denn je möglich, den färberischen Nachweis von Tuberkelbacillen in bacillenarmen Medien zu erbringen. Doch ist die Feststellung der Tuberculose auf diesem Wege nicht in allen Fällen eine einwandfreie. Insbesondere gilt dies von der Differentialdiagnose zwischen dem Tuberkelbacillus und dem Smegmabacillus, welche häufig nicht leicht $z u$ stellen ist. Aehnliches gilt in selteneren Fällen auch vom Sputum. Wir erinnern an die. Befunde von Zahn, Pappenheim, Fraenkel und Rabinowitsch, die im Sputum bei Lungengangrän säurefeste Bacillen fanden, während die Obduction keine Tuberculose ergab. Rabinowitsch gelang die Reinzüchtung dieser Bacillen, die sich für Meerschweinchen als nicht pathogen erwiesen und in die Gruppe der Buttersäurebacillen eingereiht wurden. Auch Laabs fand bei angeblich nicht tuberculösen Individuen säurefeste Stäbchen im Mundspeichel, Zungenund Zahnbelag. Schliesslich sei auf die im Wasser vorkommenden, von Antiformin nicht angreifbaren säurefesten Bacillen hingewiesen, die auch zu Fehlschlüssen Anlass geben können. Ausserdem gibt es eine ganze Anzahl von Fällen, bei denen der mikroskopische Nachweis von Tuberkelbacillen überhaupt nicht gelingt und die dennoch klinisch der Tuberculose höchst verdächtig erscheinen. Insbesondere gilt dies von serösen Exsudaten und Gelenkergüssen.

Für all die fraglichen Fälle kommt praktisch nur der biologische Weg als das einzig zuverlüssige Mittel in Betracht. Allerdings haftet der bisher meist geübten intraperitonealen Impfung trotz ihrer Zuverlässigkeit der Mangel an, dass der sichere Nachweis der specifischen Natur des geimpften Materials erst in einem Zeitraum von 6 bis 7 Wochen möglich ist.

In den letzten Jahren sind nun mannigfache Versuche angestellt worden, die auf die Beschleunigung des Nachweises der specifischen Natur des Impfmaterials hinzielten. Die Art dieser Versuche bewegt sich nach zwei Richtungen hin. Auf der einen Seite versuchte man die er- 
höhte Emptindlichkeit gewisser Organe gegen die tuberculöse Infection auszunïtzen, indem eine directe Impfung in diese Gewebe vorgenommen wurde. Auf der andern Seite nützte man die Ueberempfindlichkeit der geimpften Meerschweinchen gegen Tuberculin aus, um die tuberculöse Erkrankung des Tieres früher nachweisen zu können. Schliesslich wurde die Allergie sicher tuberculöser Tiere dazu benützt, um durch Intracutanimplung mit dem fraglichen Material eine Intracutanreaction zu erzielen (Hagemann).

In die erste Kategorie dieser Versuche wäre einzureihen die Methode von Damsch, welcher durch Impfung in die vordere Augenkammer des Kaninchens nach drei Wochen deutlich erkennbare Tuberkel auf der Iris nachweisen konnte. Dieser Wog erwies sich jedoch praktisch als wenig brauchbar, wegen der schwierigen Technik sowie wegen der bei Bestehen einer Mischinfection auftretenden Panophthalmie, die den Nachweis der specifischen Veränderungen der Iris unmöglich macht. Nattan-Larrier und Griffon haben Veerschweinchen in der Laktationsperiode das verdächtige Material in die Brustdrüsen injiciert und nach 5 bis 6 Tagen in der ansgequetschten Milch Tuberkelbacillen nachweisen können.

Die Beobachtung, dass die regionären Lymphdrüsen der Tuberculoseinfection früzeitig anheimfallen, gab Salus und Rabinowitsch Veranlassung, das verdächtige Material subcutan in die Leistengegend zu injicieren. Die geschwollenen Leistendrüsen erwiesen sich nach 3 bis 4 Wochen als tuberculös.

Die durch ein Trauma geschaffene Disposition zur schnelleren tuberculösen Erkrankung veranlasste Bloch, die Leistendrüsen vor der subcutanen, Injection zu quetschen. In den bereits nach 9 Tagen geschwollenen Drüsen konnte er in Schnittpräparaten Tuberkelbacillen nachweisen.

Die immerhin complicierte Methode des Nachweises von Tuberkelbacillen in den Serienschnitten haben Bloch und gleichzeitig Bachrach und Necker dadurch vereinfacht, dass sie die Drüsen in 15 proc. Antiformin auflösten und das Sediment mikroskopisch untersuchten. Bereits nach 10 bis 11 Tagen konnten Bachrach und Necker auf diesem Wege die tuberculöse Natur der Erkrankung in allen verdächtigen 25 Fällen nachweisen. Als Untersuchungsmaterial diente ausschliesslich Harn. Dieser so beschleunigte Nachweis von Tuberkelbacillen wäre ohne Zweifel allen bisher geübten Methoden überlegen, wenn er eben absolut sicher wäre. Die Erfahrung lehrt indes, dass die Schwellung und die centrale Erweichung der Inguinaldrüsen nicht immer specifiseh ist, da auch andere Bakterien diese Veränderungen bedingen können. Sogar die gefundenen säurefesten Bacillen können miteingebrachte, nicht specifische Erreger sein, wie dies aus den Untersuchungen ron Dieterlen hervorgeht. Schliesslich kann nach Esch die Drüsenschwellung überhaupt fehlen, besonders in Fällen, in wolchen nur äusserst minimale Mengen von Tuberkelbacillen injiciert wurden.

Auch die von Esch angegebene intracardiale Einspritzung des verdächtigen Materials hat sich nicht eingebürgert. Es scheint, dass die Technik zu schwierig ist und die Tiere häufig im Anschluss an die Impfung an Verblutung zugrunde gehen (s. Hagemann). 
Oppenheimer wählte für die Injection des infectiösen Materials die Leber, von der Erfahrung ausgehend, dass nach Einbringung von Tuberkelbacillen in die Blutbahn Leber und Milz zuerst erkranken. Seine Versuche ergaben, dass regelmässig bei Ueberimpfung von tuberkelbacillonhaltigem Harn bereits nach 16 Tagen, öfter auch schon früher bis herab zu 5 T'agen, miliare Knötchen in der Leber und Hilz nachweisbar waren, die bei der mikroskopischen Untersuchung sieh immer als Tuberkel orwiesen. Die Vorteile dieser Impfmethode bestehen in der sehr einfachen Technik, wie insbesondere in der bereits makroskopisch sichtbaren anatomischen Veränderung, die bei einiger Uebung die weitere histologische Untersuchung überflüssig macht. Zwar erseheint der sichere Nachweis der Tuberculose durch die intrabepatische Methode gegenüber der von Bachrach und Necker modificierten Blochschen Methode unter Umständen um einige Tage verzögert, dafür entfallen alle Bedenken bezüglich der Identität der Bacillen, wodurch der Nachweis ein absolut verlässlicher ist.

Aus der Oppenheimerschen Arbeit geht hervor, dass bei der intrahepatischen Impfung regelmässig auch Tuberculose der Milz bestand, die häufig ausgesprochener war als die der Leber, und manchmal sogar früher auftrat.

Diese Beobachtung im Zusammenhange mit der Errfahrung, dass bei der Obduction tuberculöser Meerschweinchen die Hilz häufig sich als am schwersten afficiert erweist, veranlasste uns, die Milz als Impfstelle zu benützen, um womöglich auf diesem Woge den Nachweis der tuberculösen Infection noch weiter abkürzen zu können. Unsere Erfahrungen haben nun in der Tat unsere Annahme insoweit bestätigt, als sich bei unseren Versuchen in der Regel nach 14 Tagen, in der Mehr»ahl der Fälle nach 10 Tagen, häufig auch schon früher, die tuberculöse Infection nachweisen liess.

Zunächst schien allerdings die Technik complicierter und zeitraubender als bei der Oppenheimerschen Methode. Denn es erscheint bei der Kleinheit der Milz unmöglich, mit einiger Sicherheit in dieselbe zu impfen, wovon wir uns in mehreren Vorversuchen überzeugen konnten. Nur durch die Laparotomie ist cine verlässliche Impfung möglich; diese gestaltet sich bei einiger Uebung äusserst einfach.

Die Bauchhöhle des narkotisierten und rasierten Meerschweinchens wird mit einem linksseitigen Flankenschnitt, der vom Rippenbogen in der Länge von etwa $5 \mathrm{~cm}$ nach abwärts geführt wird, geöffnet; dabei stellt sich.der Magen ein, welcher vorgezogen wird. An seiner grossen Curvatur hängt die etwa $2 \mathrm{~cm}$ lange $\mathrm{Mil} z$, die automatisch mit dem Magen aus der Wunde gleitet. In diese wird nun das verdüchtige Material mit einer ganz feinen und kurzen Kanüle cingespritzt. Hierbei ist nur darauf $\% u$ achten, dass die sehr dünne Milz nicht durchgestochen und dadurch das Material in die Bauchhöhle injiciert wird. Am zweckmässigsten wird hierfür ein Milzpol zwischen Daumen und Zeigefinger fixiert. Die richtig ausgeführte Injection erkennt man an dem Entstehen einer anämischen Zone, die sich scharf gegen das rote Milzgewebe absetzt. Nach Reposition der Eingeweide werden die Bauchdecken mit 
fortlaufender Naht vereinigt, die Hant mit Michelschen Klammern geschlossen und mit etwas Collodium bossen. Die Bauchhaut wird nur mit Jodtinctur bestrichen und steril abgedeckt. Die $\Lambda$ sepsis erfordert keine besondere Aufmerksamkeit. In unserer ganzen Versuchsreihe ist uns nur zu Beginn der Versuche ein einziges Tier an Peritonitis eingegangen. Ganz vereinzelt stellten sich geringe Bauchdeckeneiterungen ein, die jedoch den Gang der Versuche in keiner Weise beeinflussten. Viel mehr zu schaffen gab uns anfänglich die parenchymatöse Milzblutung. Sie trat aus der Injectionsstelle bald sehr gering, bald abundant auf, erforderte aber in den meisten Fällen keine besondere Blutstillung. Immerhin hatten wir im Anfang einige Verblutungen in die Bauchhöhle zu verzeichnen. Der Liebenswürdigkeit des Herrn Vorstandes Prof. Dr. E. F reund im hiesigen chemischen Laboratorium verdanken wir ein Präparat, durch welches wir starke Blutungen immer beherrschen konnten. Es ist dies ein Lecithinpräparat "Coagulint".

Auf die blutende Stelle wird durch einige wenige Yinuten ein mit diesem Präparate getränkter Tupfer aufgedrückt. Seit Anwendung dieses Nittels ist uns kein Tier mehr an Verblutung eingegangen. Die Blutung aus den Bauchdecken ist eine minimale und erfordert in der Regel keine Gefässunterbindung. Es empfiehlt sich, ziemlich weit seitlich, am besten links in der vorderen Axillarlinie, zu laparotomieren, um den grösseren Gefässen auszuweichen. Der ganze Eingriff gestaltet sich technisch sehr leicht, ist von einem Operateur ohne Assistenz ausführbar und davert kaum zehn Minuten. Das Instrumentarium besteht nur aus einem Scalpell, einer kleinen Schere, einer kleinen chirurgischen Pincette, kleiner stark gebogener Nadel, feiner Seide und Michelschen Klammern. Zur Injection verwenden wir eine $2 \mathrm{ccm}$ fassende Recordspritze.

Die Nenge des injicierten Materials beträgt meist $1 / 2$ bis 1 , höchstens $1 \frac{1}{2} \mathrm{ccm}$. Crössere Mengen können wegen der Kleinheit des Organs nicht injiciert werden. Zur Prüfung des Erfolges bedienten wir uns der Relaparotomie der injicierten Tiere. Dies gewährte uns den Vorteil, sehr frühzeitig nachsehen und bei negativem Befunde warten zu können. So sparten wir Material und liefen nicht Gefahr, ein nur vorläufig negatives Resultat als definitives anzusprechen. Die Relaparotomie führten wir median aus; sie wird von den meisten Tieren ebenso gut vertragen wie dic erste. In negativen Fällen haben wir hier und da auch wiederholt relaparotomiert.

Exsudate, Harnsedimente und Eiter wurden ohne jede weitere Vorbehandlung injiciert. Nur eine Anzahl von Exsudaten wurde gleich nach Entnahme mit sterilen Porzellankügelchen längere Zeit geschüttelt, um zu verhindern, dass das sich stets bildende und nicht injicierbare Fibringeriunsel die meist ohnehin sehr spärlichen Tuberkelbacillen einschliesse. Mehr Schwierigkeiten machte die Injection des Sputums. Um die von Jacoby und Meyer festgestellte schädigende Wirkung der stärker concentrierten Antiforminlösung auf die Virulenz der Bacillen zu vermeiden, haben wir verschiedene Modificationen der Vorbehandlung des Sputums versucht. Das nicht vorbehandelte Sputum ist natürlich wegen seiner Consistenz unbrauchbar, da es sich durch die unbedingt notwendigen 
feinen Canülen nicht injicieren lässt. Versuche, das Sputum durch Schütteln mit Glasperlen so weit zu homogenisieren, dass es injicierbar wird, ergaben kein Resultat. Wir bedienten uns ferner der Autolyse des Sputums, indem wir dieses 12 bis 24 Stunden im Thermostaten stehen liessen. Es gelingt auf diese Weise, jedes Sputum so weit zu verflüssigen, dass man es ohne Schwierigkeiten injicieren kann. Mit derart homogenisiertem Sputum haben wir nun eine grössere Versuchsreihe angestellt. Wir können aber diese Methode nicht empfehlen, weil uns mehr als die Hälfte der Tiere bereits am Tage nach der Injection eingegangen ist. Die Obduction ergab meistens einen ganz negativen Befund. Da im Ausstrichpräparat solcher autolysierter Sputa die übrigen Bakterien ungeheuer vermehrt sind, nehmen wir an, dass dio Tiere an acuter Sepsis eingegangen sind.

Wir gingen daher zu Versuchen mit schwachen Antiforminlösungen über. Bei 5 proc. Lösungen ergab sich der Uebelstand, dass das Homogenisieren sehr lange Zeit dauerte und sich beim Centrifugieren ein sehr mangelhaftes Sediment bildete, das oft weniger Bacillen enthielt, als das unvorbehandelte Sputum. Der Zusatz von grösseren Mengen Alkohol, der ein besseres Absetzen ermöglicht hätto, musste natürlich vermieden werden, um die Bacillen in ihrer Virulenz nicht \%u schädigen.

Wir haben nun auf folgende Weise versucht, die langdauernde Einwirksung des Antiformins auf das Sputum zu vermeiden. Wir überliessen zunächst das Sputum im Thermostat der Autolyse, schüttelten es dann mit der annähernd gleichen Menge 10- oder 15proc. Antiforminlösung gründlich durch, wobei die Homogenisierung in ganz kurzer Zeit vor sich ging. Dann wurde möglichst rasch abcentrifugiert, das Sediment in wenig physiologischer Kochsalzlösung aufgeschwemmt und injiciert. Diese Methode hat sich uns weitaus am besten bewährt, wobei die 10 proc. I.ösungen günstigere Resultate lieferten als die 15 proc.

Es seien nun die gewonnenen Resultate in tabellarischer Uebersicht angeführt.

Zunächst mögen einige Versuche mit Reinculturen folgen. Es wurde eine Aufschwemmung einer Oese Tuberkelbacillen vom Typus humanus auf $10 \mathrm{ccm}$ physiologischer $\mathrm{NaCl}-J$ Jösung als Grundaufschwemmung verwendet. Wir werden diese Aufschwemmung der Kürze halber als Normalaufsebwemmung bezeichnen.

'I'abelle l.

Versuche mit Sormalaufschwemmung.

\begin{tabular}{c|c|c|c|c}
\hline & Menge & $\begin{array}{c}\text { Datum } \\
\text { der } \\
\text { Injection }\end{array}$ & $\begin{array}{c}\text { Datum } \\
\text { der } \\
\text { Relaparotomie }\end{array}$ & E r g e b n is \\
\hline 16 & 1 & 8.10. & 16.10. & $\begin{array}{c}\text { Milz 2 fach vergrössert, 2 grosse käsige } \\
\text { Knoten; im Ausstrichpräparate reieh- } \\
\text { liche Tuberkelbacillen. Leber 0. } \\
\text { Milz: mehrere hanfkorngrosse verkäste } \\
\text { Knötchen. Leber 0. }\end{array}$
\end{tabular}


Beschleunigter Nachweis der'T'ubereulose im Tierversuch darch Milzimpfung. 279

\begin{tabular}{|c|c|c|c|c|}
\hline 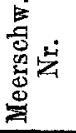 & $\begin{array}{l}\text { Menge } \\
\text { cem }\end{array}$ & $\begin{array}{l}\text { Datum } \\
\text { der } \\
\text { Injection }\end{array}$ & $\begin{array}{c}\text { Datum } \\
\text { der } \\
\text { Relaparotomie }\end{array}$ & Ergebnis \\
\hline 23 & $1 / 2$ & 9. 10. & 16. 10 . & $\begin{array}{l}\text { Milz 3 fach vergrössert, zahlreiche ver- } \\
\text { käste bis hanfkorngrosse Knötchen; } \\
\text { im Ausstrich Bacillen positiv. In } \\
\text { der Leber zahlreiche gelbe Knötchen. } \\
\text { Milz 3 fach vergrössert, durchsetzt von } \\
\text { gelben verkästen Knötchen, im Aus- } \\
\text { strich Bacillen positiv. Leber 0. }\end{array}$ \\
\hline $\begin{array}{l}26 \\
32\end{array}$ & $\begin{array}{l}1 / 2 \\
1\end{array}$ & $\begin{array}{r}9.10 . \\
13.10 .\end{array}$ & $\begin{array}{l}15.10 . \\
19.10 .\end{array}$ & $\begin{array}{l}\text { Verkäste Knötchen in Milz und Leber. } \\
\text { Milz doppelt vergrössert; an der Ein- } \\
\text { stichstelle ein grosser käsiger Knoten } \\
\text { neben mehreren kleineren. Leber } 0 . \\
\text { Ausstrich positiv. }\end{array}$ \\
\hline 37 & 1 & 18. 10. & $23 \cdot 10$. & $\begin{array}{l}\text { Milz doppelt vergrössert; enthält ver- } \\
\text { käste Knötchen. Miliare Knötchen } \\
\text { der Leber. }\end{array}$ \\
\hline 38 & $1 / 2$ & 18. 10. & 23. 10 & $\begin{array}{l}\text { Verkäste Knötchen der Milz und Leber. } \\
\text { Im Ausstrich Bacillen positiv. Leber } 0 \text {. }\end{array}$ \\
\hline 44 & 0,1 & 28. 10 & $\left\{\begin{aligned} 4 . & 11 . \\
10 . & 11\end{aligned}\right.$ & $\begin{array}{l}\text { Negativ. } \\
\text { Drei grosse käsige Knoten der Milz. } \\
\text { Bacillen positiv. }\end{array}$ \\
\hline 48 & 0,01 & 28. 10 & & $\begin{array}{l}\text { Mehrere grosse käsige Milzknoten. Im } \\
\text { Ausstrich Bacillen positiv. Leber } 0 .\end{array}$ \\
\hline 50 & 0,001 & 28. 10 . & $\left\{\begin{array}{r}8.11 \\
11.11\end{array}\right.$ & $\begin{array}{l}\text { Zwei verkäste Knoten in der Milz. } \\
\text { Leber } 0 . \text { A Asstrich positiv. }\end{array}$ \\
\hline
\end{tabular}

Aus dieser Tabelle geht wohl eindeutig hervor, dass die Menge der eingebrachten Keime den Zeitpunkt, an dem eine makroskopische Erkrankung der Milz nachweisbar ist, ausserordentlich beeinflusst, eine übrigens längst bekannte Tatsache. Bei grossen Mengen haben wir regelmässig schon in der ersten Woche, frühestens am fünften Tage, ausgesprochene tuberculöse Veränderungen nachweisen und durch positiven Bacillenfund erhärten können. Bei stärkeren Verdünnungen liess sich Tuberculose der Milz erst nach 12 bis 13 Tagen constatieren.

Tabelle II.

Autolysiertes Nativsputum in die Milz.

\begin{tabular}{|c|c|c|c|c|c|}
\hline 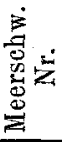 & $\begin{array}{c}\text { Menge des } \\
\text { injicierten } \\
\text { Sputums } \\
\text { ecm }\end{array}$ & $\begin{array}{c}\text { Bacillen- } \\
\text { gehalt }\end{array}$ & $\begin{array}{c}\text { Datum der } \\
\text { Injection }\end{array}$ & $\begin{array}{l}\text { Datum der } \\
\text { Rela- } \\
\text { parotomie }\end{array}$ & Ergebais \\
\hline 110 & $1 / 2$ & Reichlich & 27. 11 . & 10. 12 . & $\begin{array}{l}\text { Zwei grosse käsige Milzknoten. } \\
\text { Im Ausstrich Bacillen positiv. }\end{array}$ \\
\hline 112 & $1 / 2$ & $\begin{array}{l}\text { Mässig, } \\
\text { Reichlich }\end{array}$ & 27. 11 . & 15. 12 . & $\begin{array}{l}\text { Mässige Tuberculose der Milz } \\
\text { und Leber. }\end{array}$ \\
\hline 117 & $1 / 2$ & Reichlich & 2,12 . & 16. 12 . & $\begin{array}{l}\text { Tuberculose der vergrösserten } \\
\text { Milz. Leber } 0 \text {. }\end{array}$ \\
\hline 134 & 1 & $\begin{array}{l}\text { Mässig, } \\
\text { Reichlich }\end{array}$ & 4. 12 . & 16. 12 . & Tuberculose der Milz. \\
\hline 104 & $1 / 2$ & Spärlich & 24. 11 . & $\begin{array}{c}9.12 . \\
\text { Spontanexitus }\end{array}$ & $\begin{array}{l}\text { Milzabscess an der Injections- } \\
\text { stelle. Fraglich. }\end{array}$ \\
\hline 103 & $1 / 2$ & Reichlich & 24. 11 & $\begin{array}{l}\text { 2. } 12 . \\
\text { Narkosetod }\end{array}$ & Negativ (nach 8 Tagen). \\
\hline
\end{tabular}

Sieben Tiere 24 Stunden bis 3 Tage nach der Injection eingegangen. 
Hs wurden somit im ganzen 13 Tiere mit autolysiertem Nativsputum in die Milz geinupft. Bei vier Tieren ergab die nach 12, 13, 14 und 18 Tagen vorgenommene Relaparotomie ein positives Resultat. Ein Tier zeigte nach 8 Tagen keine Tuberculose; infolge Narkosetodes während der Relaparotomie konnte kein ondgiltiges Resultat erzielt werden.

Fraglich ist das Ergebnis bei Tier 104, da durch ein Uebersehen der Eiter des Milzabscesses nicht auf Tuberkelbacillen gefärbt wurde.

So günstig nun diese Resultate auch sind, ist die Methode wegen des grossen Verlustprocentsatzes (54 pCt.) nicht empfehlenswert.

Tabellc III a.

Autolysiertes Antiforminsputum in die Milz.

A. 10 procentig.

\begin{tabular}{|c|c|c|c|c|c|}
\hline 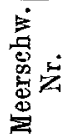 & $\left|\begin{array}{c}\text { Menge des } \\
\text { injicierten } \\
\text { Sputums } \\
\text { ccm }\end{array}\right|$ & $\begin{array}{l}\text { Bacillen- } \\
\text { gehalt }\end{array}$ & $\begin{array}{l}\text { Datum der } \\
\text { Injection }\end{array}$ & $\begin{array}{l}\text { Datum der } \\
\text { Rela- } \\
\text { parotomie }\end{array}$ & Ergebas \\
\hline 66 & 1 & Spärlich. & 5. 11. & 12. 11. & $\begin{array}{l}\text { Negativ. } \\
\text { Tuberculose der Milz. }\end{array}$ \\
\hline 67 & 1 & $\begin{array}{l}\text { Mässig } \\
\text { reichlich. }\end{array}$ & 5. 11. & 12. 11 . & Negativ. Narkosetod. \\
\hline 68 & 1 & $\begin{array}{l}\text { Mässig } \\
\text { reichlich. }\end{array}$ & 5. 11. & 12. 11. & $\begin{array}{l}\text { Ein lëisiger Knoten der ver- } \\
\text { grösserten Milz. Im Ausstrich } \\
\text { Bacillen positiv. }\end{array}$ \\
\hline 71 & 1 & $\begin{array}{l}\text { Mässig } \\
\text { reichlich. }\end{array}$ & 5. 11 . & 12. 11 . & $\begin{array}{l}\text { Langer käsiger Milzknoten, da- } \\
\text { neben kleinere verkäste. Im } \\
\text { Ausstricls Bacillen positiv. }\end{array}$ \\
\hline 73 & 1 & $\begin{array}{l}\text { Sehr } \\
\text { spärlich. }\end{array}$ & 6. 11 . & $\begin{array}{l}\text { 13. } 11 . \\
\text { 17. } 11 .\end{array}$ & $\begin{array}{l}\text { Negativ. } \\
\text { Spontanexitus: Negativ. }\end{array}$ \\
\hline 82 & 1 & $\begin{array}{l}\text { Sehr } \\
\text { spärlich. }\end{array}$ & 17. 11 . & $\begin{aligned} 28 . & 11 . \\
2 . & 12 . \\
\text { 5. } & 12 .\end{aligned}$ & $\begin{array}{l}\text { Negativ. } \\
\text { Fragliche Knötchen in der Leber. } \\
\text { Milz 0. } \\
\text { Exitus: Spärliche Tuberculose } \\
\text { der Milz und Leber. }\end{array}$ \\
\hline 93 & 1 & Spärlich. & 21. 11. & 13. 12. & $\begin{array}{l}\text { Zwei grosse käsige Milzknoten. } \\
\text { Tbc--Bacillen im Ausstricl } \\
\text { positiv. }\end{array}$ \\
\hline 106 & $1 / 2$ & $\begin{array}{l}\text { Mässig } \\
\text { reichlich. }\end{array}$ & 26. 11. & 15. 12. & $\begin{array}{l}\text { Grossknotige The. der stark ver- } \\
\text { grösserten Milz: Miliare der } \\
\text { Leber. }\end{array}$ \\
\hline 107 & $1 / 2$ & $\begin{array}{l}\text { Mässig } \\
\text { reichlich. }\end{array}$ & 26.11 . & 15. 12. & $\begin{array}{l}\text { Grosse käsige Knoten der stark } \\
\text { vergrösserten Milz. Leber } 0 \text {. }\end{array}$ \\
\hline 108 & $1 / 2$ & Spärlich. & 24.11. & 12. 12. & $\begin{array}{l}\text { Grosse Tbe.-Knoten der Milz, } \\
\text { miliare in der Leber. }\end{array}$ \\
\hline
\end{tabular}

Es wurden in ganzen 10 Tiere mit 10proc. autolysiertem Antiforminsputum geimpft. Positive Resultate ergaben sich am 6., 7., 13., 18., 19., 19. und 22. Tage. Die Tiere 93, 106, 107, 108 konnten aus äusseren Umständen erst zu so späten Terminen relaparotomiert werden; sie zeigten aber schon so weit fortgeschrittene tuberculöse Veränderungen der Milz, dass sic sicher schon mehrere Tage früher ein positives Resultat ergeben hätten.

Negative Befunde hatten wir am 7., 7., 8., 11. und 11. Tage. Das T'ier 67 zeigte nach 7 Tagen keine Tuberculose; infolge Narkosetodes während der Relaparotonie konnte kein endgiltiges Resultat erziclt werden. 
Bei Tier 82 ergab sich nach 11 Tagen ein negatives, nach 15 Tagen ein fragliches, nach 18 Tagen ein sicher positives Resultat. Der Ausfall des Versuchs bei diesem Tier beweist die Richtigkeit unseres Vorgehens, die Tuberculose nicht durch eine eventuell zu frühzeitige Obduction, sondern durch Relaparotomie nachzuweisen. Bei Anwendung der ersten Methode wäre das Resultat des Tierversuches unrichtig gewesen.

T'a bello III b.

Autolysiertes Antiforminspatum in die Milz.

B. 15 procentig.

\begin{tabular}{|c|c|c|c|c|c|}
\hline 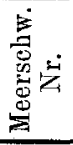 & \begin{tabular}{|} 
Menge des \\
injicierten \\
Sputums \\
ccm \\
\end{tabular} & $\begin{array}{l}\text { Bacillen- } \\
\text { gehalt }\end{array}$ & $\begin{array}{l}\text { Datum der } \\
\text { Injection }\end{array}$ & $\begin{array}{l}\text { Datum der } \\
\text { Rela- } \\
\text { parotomie }\end{array}$ & Ergebnis \\
\hline$\dot{6}$ & $1 / 2$ & Mässig & 25. 9 . & $\begin{array}{l}22.10 . \\
\text { Spontan- } \\
\text { exitus. }\end{array}$ & $\begin{array}{l}\text { Diffuse Tbc. der Milz, Leber, } \\
\text { Kiralyfische Drüsen, Perito- } \\
\text { neum-Tbc. }\end{array}$ \\
\hline 8 & 1 & Reichlich. & 1. 10. & 15.10 & $\begin{array}{l}\text { Milz } 4 \text { fach vergrössert, entbält } \\
\text { zahlreiche kleinere, vereinzelte } \\
\text { grössere, fast durehweg ver- } \\
\text { käste Knoten. Leber: mässig }\end{array}$ \\
\hline 11 & 2 & Reicblich. & 3. 10. & 19. 10. & $\begin{array}{l}\text { Milz } 3 \text { fach vergrössert, sehr zahl- } \\
\text { reiche Knötchen. Kiralyfische } \\
\text { Drüsen (verkäst). }\end{array}$ \\
\hline 12 & 1 & Mässig. & 3. 10 & 16. 10. & $\begin{array}{l}\text { Milz } 4 \text { fach vergrössert, zahlreiche } \\
\text { verkäste Knoten. Leber } 0 \text {, } \\
\text { fettig degeneriert. }\end{array}$ \\
\hline 14 & 1 & Zahlreich. & 4. 10 . & 16. 10. & $\begin{array}{l}\text { Ein Knötchen in der Milz, } \\
\text { 20. 10. Spontanexitus: auch } \\
\text { Leber-Tbe. }\end{array}$ \\
\hline 58 & 1 & Spärlich. & 30. 10. & $\begin{array}{l}\text { 8. } 11 . \\
11.11 .\end{array}$ & $\begin{array}{l}\text { Negativ. } \\
\text { Zwei hanfkorngrosse verkäste } \\
\text { Milzknoten. Leber } 0 . \text { Aus- } \\
\text { strich positiv. }\end{array}$ \\
\hline 59 & 1 & $\begin{array}{l}10 \text { fach } \\
\text { verdünntes } \\
\text { Sputum v. } \\
\text { Vers } 58\end{array}$ & 30. 10. & $\begin{array}{r}\text { 8. } 11 . \\
12.11 .\end{array}$ & $\begin{array}{l}\text { Negativ. } \\
\text { Käsige Knoten in der Milz. } \\
\text { Leber } 0 .\end{array}$ \\
\hline 60 & 1 & $\begin{array}{l}100 \text { fach } \\
\text { verdünntes } \\
\text { Sputum v. } \\
\text { Vers. } 58 .\end{array}$ & 30. 10 . & $\begin{aligned} \text { 12. } & 11 . \\
\text { 1. } & 12 .\end{aligned}$ & $\begin{array}{l}\text { Negativ. } \\
\text { Ausgebreitete Tbc. der Milz, } \\
\text { Leber und Peritoneum. }\end{array}$ \\
\hline 61 & 1 & $\begin{array}{l}1000 \text { fach } \\
\text { verdünntes } \\
\text { Sputum v. }\end{array}$ & 30. 10 . & $\begin{array}{l}\text { 12. } 11 . \\
23.11 .\end{array}$ & $\begin{array}{l}\text { Negativ. } \\
\text { Käsige Knoten der Milz. }\end{array}$ \\
\hline 62 & 1 & Spärlich. & 31. 10 . & $\begin{array}{r}8.11 . \\
11.11 .\end{array}$ & $\begin{array}{l}\text { Negativ. } \\
\text { Miliare Tbc. der Milz. }\end{array}$ \\
\hline
\end{tabular}

Es wurden im ganzen 10 Tiere mit 15 proc. autolysiertem Antiforminsputum geimpft. Positive Resultate ergaben sich am 12., 12., 12., 13., 13., 14., 16., 23. (27. und 30.) Tage. Das Tier 6, das aus der Zeit unserer allerersten Versuche stammt, ging nach 27 Tagen spontan ein, cine Relaparotomie wurde nicht gemacht; es lässt sich daher nicht abschätzen, wann das Tícr bereits makroskopische Tuberculose zeigte. Bei Tier $\mathrm{Nr}$. 60, das mit 100 fach verdüntem Sputum geimpft wurde, wurde es übersehen, das Tier zu einem früheren Termin zu relaparotomieren. 
Doch muss man annehmen, dass es zumindest nach 23 Tagen tuberculös erkrankt war, weil das Tier $\mathrm{Nr} .61$, das mit einer noch zehnmal stärkeren Verdünnung geimpft wurde, bereits um diese Zeit deutliche Tuberculose der Milz zeigte.

Die negativen Resultate stammen vom 8., 9., 9., 12., 12. Tage.

Vergleicht man die Tabellen A und B, so scheinen die Versuche mit 10 proc. Antiforminverdünnung günstigere Resultate zu liefern, als die mit 15 pros. Wir lassen es dahingestellt, ob diese Differenz auf die schädigende Wirkung der stärker concentrierten Antiforminlösung zurüekzuführen ist. Um dies zu entscheiden, jst unsere Versuchsreihe zu klein.

T a b e I 10 IV.

Exsudate.

\begin{tabular}{|c|c|c|c|c|c|}
\hline 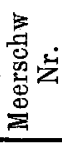 & \begin{tabular}{|c|} 
Menge des \\
injicierten \\
Exsudats \\
cem
\end{tabular} & Chemisch-mikroskospischer Befund & $\begin{array}{l}\text { Datum } \\
\text { der } \\
\text { Injection }\end{array}$ & $\begin{array}{l}\text { Datum } \\
\text { der } \\
\text { Laparo- } \\
\text { tomie }\end{array}$ & Ergeb is \\
\hline 13 & 1 & $\begin{array}{l}\text { (Pleura) serös. Lymphocyten, spärlich } \\
\text { Leukocyten, keine Bakterien, keine } \\
\text { Tbc-Bacillen, Cultur steril, Rivalta } \\
\text { positiv. }\end{array}$ & 4. 10 . & 16. 10 & $\begin{array}{l}\text { Kleine verkäste Knötchen der Milz } \\
\text { (Ausstrich positiv), spärlichste } \\
\text { gelbe Knötchen der Leber (nicht } \\
\text { weiter untersucht). }\end{array}$ \\
\hline 34 & $11 / 2$ & (Pleura). Befund wie bei Nr. 13. & 13. 10 . & $\begin{aligned} 20 . & 10 . \\
7 . & 11\end{aligned}$ & $\begin{array}{l}\text { Negativ. } \\
\text { Diffuse Tbc. d. Milz u. Leber, verkäste } \\
\text { Kiralyfidrüsen u. ad portam hepat. }\end{array}$ \\
\hline 56 & 1 & $\begin{array}{l}\text { (Pleura) hämorrhagisch. Mikroskopisch } \\
\text { verfettete Leukocyten u. Epithelzellen, } \\
\text { Erythrocyten und Erythrocytenreste, } \\
\text { spärliche Lymphocyten, Rivalta pos., } \\
{ }^{*} \text { Cultur steril, keine Tbc.-Bacillen. }\end{array}$ & 30. 10 . & $\begin{array}{l}\text { 13. } 11 \\
25.11\end{array}$ & $\begin{array}{l}\text { Negativ. } \\
\text { Negativ. Klinisch Bronchuscarcinom } \\
\text { oder Tbc. Später vorgenommeno } \\
\text { Probeexcision einer Drüse ergab } \\
\text { Adenocarcinom. }\end{array}$ \\
\hline 64 & 1 & $\begin{array}{l}\text { (Kniegelenkerguss) serös. Im Sediment } \\
\text { Lymphocyten und Leukocyten, keine } \\
\text { Tbc.-Bacillen. }\end{array}$ & 4. 11 . & $\begin{array}{l}\text { 19. } 11 \\
28.11\end{array}$ & $\left\{\begin{array}{l}\text { Negativ. Klinisch und therapeutisch } \\
\text { ergab sich Gonitis gonorrhoica. }\end{array}\right.$ \\
\hline 69 & 1 & $\begin{array}{l}\text { (Pleura) hämorrhagisch. Mikroskopisch } \\
\text { Erythrocyten, spärlich Jympho- und } \\
\text { Leukocyten, spärlich Tbc--Bacillen. }\end{array}$ & 5. 11 . & 12. 11 & $\begin{array}{l}\text { Grössere und kleinere verkäste Knöt- } \\
\text { chen der Milz, Ausstrich positiv. }\end{array}$ \\
\hline 76 & 1 & $\begin{array}{l}\text { (Peritoneum) serös. Spärlich Leuko- } \\
\text { und Lymphocyten, Rivalta positiv, } \\
\text { keine Tbc-Bacillen. }\end{array}$ & 11. 11. & $\begin{array}{ll}28 . & 11 \\
12 . & 12 \\
18 . & 12\end{array}$ & $\left\{\begin{array}{l}\text { Negativ. Klinische Diagnose: Tbe. } \\
\text { pleurae et peritonei. Obductions- } \\
\text { diagnose: Hepatitis interst., Pfort- } \\
\text { aderthrombose, Ascites, keine Tbc. }\end{array}\right.$ \\
\hline 79 & $11 / 2$ & $\begin{array}{l}\text { (Pleura) serös. Im Sediment zahlreiche } \\
\text { Erythrocyten, spärlich Lympho- und } \\
\text { Leukocyten, keine Tbe.-Bacillen. }\end{array}$ & 17. 11 . & $\begin{array}{l}\text { 2. } 12 . \\
\text { 5. } 12\end{array}$ & $\begin{array}{l}\text { Negativ. } \\
\text { Zwei grosse Tbc.-Knoten im Milz- } \\
\text { parenchym, Ausstr. pos., Ieber } 0 .\end{array}$ \\
\hline 96 & 1 & $\begin{array}{l}\text { (Pleura) serös. Reichliche Lympho- } \\
\text { cyten, keine Bacillen. }\end{array}$ & 22.11. & $\begin{array}{l}\text { 13. } 12 . \\
22.12 .\end{array}$ & $\begin{array}{l}\text { Negativ. } \\
\text { Grössere käsige Knoten der Milz, } \\
\text { spärl. verkäste Knötchen d. Jueber. }\end{array}$ \\
\hline 101 & 1 & $\begin{array}{l}\text { (Pleura) scrös. Reichlich Lympho- } \\
\text { cyten, spärlich Erythrocyten, steril, } \\
\text { keine Tbc.-Bacillen. }\end{array}$ & 22. 11 . & 12. 12. & $\begin{array}{l}\text { Grosser käsiger Milzknoten, Leber } 0 \text {, } \\
\text { Ausstrich positiv. }\end{array}$ \\
\hline 115 & 1 & $\begin{array}{l}\text { (Pleura) serös. Spärlich Lymphocyten, } \\
\text { reichliche Leukocyten, steril, keine } \\
\text { Tbc.-Bacillen. } \\
\text { (Pleura) serös. Spärliche Lymphocyten, } \\
\text { reichl. Endothelzellen u. Ery throcyten. }\end{array}$ & 28. 11 . & $\begin{array}{l}\text { 12. } 12 \\
\text { 14. } 12 . \\
\text { Exitus } \\
\text { 16. } 12 .\end{array}$ & $\begin{array}{l}\text { Negativ. } \\
\text { Zwei käsige Milzknoten, Ausstrich } \\
\text { positiv. } \\
\text { Grosser Tuberkel der Milz. Leber } \\
\text { negativ. }\end{array}$ \\
\hline 148 & 1 & $\begin{array}{l}\text { (Peritoneum) serös. Keine Bacillen, } \\
\text { spärlich Lymphocyten, Rivalta positiv. }\end{array}$ & 22,12 . & 5. 1. & $\begin{array}{l}\text { Negativ. Klinische Diagnose: Tbe. } \\
\text { pleurae et peritonei. Obductions. } \\
\text { diagnose: Carcinoma ventr., Car- } \\
\text { cinomatosis pleurae et peritonei. }\end{array}$ \\
\hline 150 & 1 & $\begin{array}{l}\text { (Ploura) serös. Keine Bacillen, Erythro- } \\
\text { und Lymphocyten. Rivalta positiv. }\end{array}$ & 26.12 . & 5. 1. & $\begin{array}{l}\text { Negativ. Von demselben Fall wie } \\
\text { Nr. } 148 \text {. }\end{array}$ \\
\hline 152 & 1 & $\begin{array}{l}\text { (Pleura) serös. Reichlich Lympho- und } \\
\text { Erythrocyten, keine Tbc--Bacillen. }\end{array}$ & 30.12 . & 5. 1 . & $\begin{array}{l}\text { Tbc.-Knoten der Milz und Leber, } \\
\text { Ausstrich positiv. }\end{array}$ \\
\hline
\end{tabular}


Von den 14 untersuchten Exsudaten scheiden für die Beurteilung der Methode zunächst 5 aus, die bei andauernd negativem Befund sich klinisch bzw. autoptisch als nicht tuberculös erwiesen. Die übrigen Exsudate ergaben am 7., 14, 15., 21. Tage negativen Befund, dagegen am 6., 7., 12, 15., 16., 18., 20., 25. und 30. Tage positive Resultate. Die Ergebnisse sind hier begreiflicherweise nicht so günstig wie bei den Sputumversuchen. Die Exsudate sind ja derart bacillenarm, dass der färberische Nachweis in der Regel nicht gelingt. In der Tat fanden wir nur einmal spärliche Bacillen. Dieser Fall hat auch sehr früh, nämlich schon nach 7 Tagen, positiven Befund ergeben.

Ls sei hier noch hervorgehoben, dass die Tuberkelknötchen manchmal nur im Parenchym sitzen und bei der blossen Inspection nicht nachweisbar sind. Dies kann zu Irrtümern Anlass geben, wie uns ein solcher wahrscheinlich bei Meerschweinchen Nr. 79 unterlaufen ist. Bei Meerschweinchen Nr. 96 wissen wir keine Ursache für das späte Auftreten der Milztuberculose. Dagegen zeigt der Befund bei Tier Nr. 34, dass wir mit der zweiten Relaparotomie viel zu lange gewartet haben. - Im Anschluss daran sejen zwoi Versuche mit Lumbalpunctaten wiedergegeben:

1. Meerschreinchen Nr. 57 crhält am $30.10 .131 \mathrm{ccm}$ verdächtiges Lumbalpunctat (hoher Druck, leicht getrübt; im Sediment Lympho- und Leukocyten in gleicher Menge, keine Tuberlelbacillen) intralienal. Am 13. und 17.11. negativer Befund. Die Obduction des Pat. ergibt allgemeine Miliartuberculose und circumscrip te tuberculöse Meningitis über den linken Centralwindungen. An der llirnbasis waren keine meningitischen Veränderungen nachweisbar.

2. Meerschweinchen Nr. 113 erhält am 27.11.13 $1 \mathrm{ccm}$ klares Lumbalpunctat (im Sediment Leukocyten und Lymphocyten sehr spärlich, hoher Druck) intralienal. Am 15. und 22. 12. negativer Befund. Klinisch bestand ursprünglich Verdacht auf tuberculöse Meningitis, der sich jedoch später nicht bestätigte.

Die Kranke genas in kurzer Zeit röllig.

Tabelle V.

Verdäehtiger Harn.

\begin{tabular}{|c|c|c|c|c|c|}
\hline 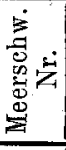 & Harnbefund & \begin{tabular}{|c|} 
Menge \\
com
\end{tabular} & $\begin{array}{l}\text { Datum der } \\
\text { Injection }\end{array}$ & $\begin{array}{c}\text { Datum der } \\
\text { Laparo- } \\
\text { tomie }\end{array}$ & Ergeb is \\
\hline 114 & $\begin{array}{l}\text { Klar. Im Sediment ver- } \\
\text { einz. Leukocyten, keine } \\
\text { säurefesten Stäbchen. }\end{array}$ & 1 & 27. 11. & 10.12 & $\begin{array}{l}\text { Zwei käsige Milzknoten. } \\
\text { Ausstrich positiv. }\end{array}$ \\
\hline 122 & $\begin{array}{l}\text { Durch Ureterenbatheter } \\
\text { entnommen, leicht } \\
\text { hämorrhagisch, im Se- } \\
\text { dimentganz vereinzelte }\end{array}$ & 1 & 29.11 . & 10. 12. & $\begin{array}{l}\text { Zwei käsige Milzknoten. } \\
\text { Ausstrich positiv. }\end{array}$ \\
\hline 123 & $\begin{array}{l}\text { säurefeste Stäbchen. } \\
\text { Klar, ohne Tbc.-Bacillen, } \\
\text { sehr spärliche Leuko- } \\
\text { cyten. }\end{array}$ & 1 & 29. 11. & $\begin{array}{l}10.12 \\
18.12 \\
24.12\end{array}$ & $\left\{\begin{array}{l}\text { Negativ. Intraperito- } \\
\text { nealer Parallelvers. v. } \\
\text { 29.11.am28.12.negativ. } \\
\text { Intrahepatisch. Vers. v. } \\
\text { 29.11.am27.12.negativ. }\end{array}\right.$ \\
\hline 137 & $\begin{array}{c}\text { Klar, kein Sediment, } \\
\text { keine Tbc.-Bacillen. }\end{array}$ & 1 & 9. 12 . & $\begin{array}{rr}20 . & 12 . \\
27 . & 12 . \\
5 . & 1 .\end{array}$ & $\left\{\begin{array}{l}\text { Negativ. Intraperito- } \\
\text { nealer Parallelversuch } \\
\text { vom } 9.12 . \text { am } 23.12 . \\
5.1 . \text { u. 20. 1. negativ. }\end{array}\right.$ \\
\hline 144 & $\begin{array}{l}\text { Klar, sehr spärliche Leu- } \\
\text { kocyten, keine Tbo.- } \\
\text { Bacillen. }\end{array}$ & 1 & 19. 12. & 5. 1. & $\begin{array}{l}\text { Negativ, tot. Intraperito- } \\
\text { nealer Parallelversuch } \\
\text { v.19.12.am3.2. negativ. }\end{array}$ \\
\hline
\end{tabular}


Zur Untersuchung gelangten wegen Mangels an Material nur sieben Harne, von wolchen drei nach allen Methoden auch nach langer Zeit ein negatives Resultat ergaben, daher für die Beurteilung ausscheiden.

Tabelle VIa.

Intralienal.

\begin{tabular}{|c|c|c|c|c|}
\hline 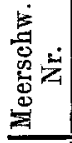 & Impfmaterial. & $\begin{array}{l}\text { Datum der } \\
\text { Impfung }\end{array}$ & $\begin{array}{c}\text { Datum der } \\
\text { Laparo- } \\
\text { tomie }\end{array}$ & Ergebnis. \\
\hline 37 & $\begin{array}{l}1 \text { ccm Normalauf- } \\
\text { schwemmung. } \\
1 / 2 \text { cem Normalauf- } \\
\text { schwemmung. }\end{array}$ & 18. 10. & 23.10 & $\begin{array}{l}\text { Milz doppelt vergrössert, ent- } \\
\text { hält stecknadelkopfgrosse ver- } \\
\text { käste Knötchen, miliare Knöt- } \\
\text { chen der Leber. } \\
\text { Verkäste Knötchen der Milz } \\
\text { und Leber. }\end{array}$ \\
\hline 44 & $\begin{array}{l}0,1 \mathrm{ccm} \text { Normalauf- } \\
\text { schwemmung. }\end{array}$ & 28. 10. & $\begin{array}{l}\text { 4. } 11 . \\
\text { 10. } 11\end{array}$ & $\begin{array}{l}\text { Negativ. } \\
\text { Drei grosse käsige Knötchen } \\
\text { der Milz. Bacillen im Aus- } \\
\text { strich positiv. Leber 0. } \\
\text { Mebrere grosse käsige Milz- } \\
\text { knoten. Ausstrich positiv. } \\
\text { Leber 0. }\end{array}$ \\
\hline 50 & $\begin{array}{l}0,001 \mathrm{ccm} \text { Normalauf- } \\
\text { schwemmung. }\end{array}$ & 28.10 . & $\begin{array}{l}\text { 8. } 11 \\
\text { 11. } 11\end{array}$ & $\begin{array}{l}\text { Negativ. } \\
\text { Zwei verkäste Milzknoten. Aus- } \\
\text { strich positiv. Leber } 0 .\end{array}$ \\
\hline 71 & $\begin{array}{l}1 \text { ccm } 10 \text { proc. Antifor- } \\
\text { minsputum mit mässig } \\
\text { reichlich Bacillen. }\end{array}$ & 6. 11. & 12. 11 & $\begin{array}{l}\text { Langer käsiger Milzknoten, da- } \\
\text { neben kleinere verkäste. Aus- } \\
\text { strich positiv. }\end{array}$ \\
\hline 73 & $\begin{array}{l}1 \text { ccm } 10 \text { proc. Antifor- } \\
\text { minsputum mit sehr } \\
\text { spärlichen Bacillen. }\end{array}$ & 6. 11 . & $\begin{array}{c}13.11 \\
\text { 17. } 11 . \\
\text { Spontantod. }\end{array}$ & Negativ. \\
\hline 96 & $\begin{array}{l}1 \text { com Pleuraexsudat, } \\
\text { serös, roichlich Lym- } \\
\text { phoyten, keine } \mathrm{Ba}- \\
\text { cillen. }\end{array}$ & 22.11 & $\begin{array}{l}13.12 \\
22.12\end{array}$ & $\begin{array}{l}\text { Negativ. } \\
\text { Grössere käsige Knoten der Milz, } \\
\text { spärliche verkäste Knötchen } \\
\text { der Leber. }\end{array}$ \\
\hline 15 & $\begin{array}{l}1 \text { cem Plouraexsudat, } \\
\text { serös, spärl. Lympho- } \\
\text { cyten, reichl. Leuko- } \\
\text { cyten, steril, keine } \\
\text { Tbc.-Bacillen. }\end{array}$ & 28. 11. & $\begin{array}{l}\text { 12. } 12 \\
\text { 14. } 12\end{array}$ & $\begin{array}{l}\text { Negativ. } \\
\text { Zwei käsige Milzknoten. Aus- } \\
\text { strich positiv. }\end{array}$ \\
\hline 22 & $\begin{array}{l}1 \text { com durch Ureteren- } \\
\text { lsatheter entleert, } \\
\text { leicht hämorrhagi- } \\
\text { scher Urin, im Sedi- } \\
\text { ment ganz vereinzelte } \\
\text { säurefeste Stäbchen. }\end{array}$ & 10. 12 & 10. 12 & $\begin{array}{l}\text { Zwei käsige Milzknoten. Aus- } \\
\text { strich positiv. }\end{array}$ \\
\hline 123 & $\begin{array}{l}1 \mathrm{ccm} \text { klarer Urin, sehr } \\
\text { spärliche Leukocyten. } \\
\text { keine Tbc.-Bacillen. }\end{array}$ & 29.11. & $\begin{array}{l}\text { 10. } 12 . \\
18.12 . \\
24.12\end{array}$ & Negativ. \\
\hline
\end{tabular}

Es sei ausdrücklich hervorgehoben, dass wir zur Herstellung völig gleicher Versuchsbedingungen das Impfmaterial in eine $2 \mathrm{ccm}$ Spritze aufzogen und davon je $1 \mathrm{ccm}$ injicierten. Auch haben wir im Gegensatz zu Oppenheimer auch für die Jeberimpfung die Tiere laparotomiert. 
Zwei Harne ergaben bereits am 13. und 11. Tage Tuberculose der Milz. Um nun festzustellen, ob die Impfung in die Milz früher ein positives Resultat ergibt, als die in die Leber, haben wir eine Reibe von Parallelversuchen angestellt.

Intrahepatal.

T a be 11 e VIb.

\begin{tabular}{|c|c|c|c|c|}
\hline 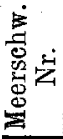 & Impfmaterial & $\begin{array}{l}\text { Datum der } \\
\text { Impfung }\end{array}$ & $\begin{array}{c}\text { Datum der } \\
\text { Laparo- } \\
\text { tomie }\end{array}$ & Ergebnis. \\
\hline 39 & $\begin{array}{l}1 \mathrm{ccm} \text { Normalauf- } \\
\text { schwemmung. }\end{array}$ & 18. 10 . & $\begin{aligned} 23 . & 10 . \\
5 . & 11 .\end{aligned}$ & $\begin{array}{l}\text { Negativ. } \\
\text { Milz 4- bis } 5 \text { fach vergrössert, } \\
\text { durehsetzt von massenhaften } \\
\text { Tbc.-Knoten, ebensodie Leber. }\end{array}$ \\
\hline 40 & $\begin{array}{l}1 / 2 \mathrm{ccm} \text { Normalauf- } \\
\text { schwemmung. }\end{array}$ & 18. 10 . & 23. 10 . & $\begin{array}{l}\text { An der Impfstelle der Leber im } \\
\text { Parenchym käsiger Eiter. Aus- } \\
\text { strich positiv, sonst } 0 \text {. }\end{array}$ \\
\hline 46 & $\begin{array}{l}0,1 \mathrm{ccm} \text { Normalauf- } \\
\text { sehwemmung. }\end{array}$ & 28. 10 . & $\begin{aligned} \text { 4. } & 11 . \\
11 . & 11 .\end{aligned}$ & $\begin{array}{l}\text { Negativ. } \\
\text { Zahlreich verkäste Knötchen der } \\
\text { Milz und Leber. }\end{array}$ \\
\hline 47 & $\begin{array}{l}0,01 \mathrm{ccm} \text { Normalauf- } \\
\text { schwemmung. }\end{array}$ & 28. 10 . & $\begin{array}{l}\text { 11. } 11 . \\
\text { 24. } 11 .\end{array}$ & $\begin{array}{l}\text { Milz stark rergrössert. Kliniseh } \\
\text { verdächtige Knöteben der Milz } \\
\text { und Leber. } \\
\text { Grosse Tbc.-Knoten in Milz und } \\
\text { Leber }\end{array}$ \\
\hline 49 & $\begin{array}{l}0,001 \mathrm{ccm} \text { Normalauf- } \\
\text { schwemmung. }\end{array}$ & 28. 10 . & $\begin{array}{l}\text { 11. } 11 . \\
1.12 \\
\text { getötet }\end{array}$ & $\begin{array}{l}\text { Negativ. } \\
\text { Spärliche Tbc.-Knoten d. Milz(!). } \\
\text { Leber } 0 \text {. }\end{array}$ \\
\hline 72 & $\begin{array}{l}1 \text { cem } 10 \text { proc. Antifor- } \\
\text { minsputum mit mässig } \\
\text { reichlich Bacillen. }\end{array}$ & 6. 11 . & 12.11 . & $\begin{array}{l}\text { In der Leber an der Impfstelle } \\
\text { ein grössererverkäster Knoten. } \\
\text { Ausstrich positiv. In der Milz } \\
\text { mehrere verkäste Knötchen. }\end{array}$ \\
\hline 74 & $\begin{array}{l}1 \text { cem } 10 \text { proc. Antifor- } \\
\text { minsputum mit sehr } \\
\text { spärlichen Bacillen. }\end{array}$ & 6. 11 . & $\begin{array}{l}\text { 13. } 11 . \\
17.11 \\
\text { getötet }\end{array}$ & Negativ. \\
\hline 100 & $\begin{array}{l}1 \text { ccm Pleuraexsudat, } \\
\text { serös, reichlich Lym- } \\
\text { phocyten, keine Ba- } \\
\text { eillen. }\end{array}$ & 22. 11 . & $\begin{array}{l}\text { 13. } 12 . \\
23.12 . \\
\text { getötet }\end{array}$ & Negativ. \\
\hline 118 & $\begin{array}{l}1 \text { cem. Pleuraexsudat, } \\
\text { serös, spärl, Lympho- } \\
\text { cyten, reichl. Leuko- } \\
\text { cyten, steril, keine } \\
\text { Tbe.-Bacillen. }\end{array}$ & 28. 11 . & $\begin{array}{l}\text { 10. } 12 . \\
\text { 18. } 12 . \\
\text { getötet }\end{array}$ & $\begin{array}{l}\text { Grosse verkäste Knötchen In der } \\
\text { Leber. Milz negativ. } \\
\text { Neben Lebertbc. Zwei verkäste } \\
\text { Knoten in der Milz. }\end{array}$ \\
\hline 125 & $\begin{array}{l}1 \text { ccm durch Ureteren- } \\
\text { katheter entleert, } \\
\text { leicht hämorrhagi- } \\
\text { scher Urin, im Sedi- } \\
\text { ment ganz vereinzelte } \\
\text { säurefeste Stäbchen. }\end{array}$ & 29. 11 . & $\begin{array}{l}\text { 10. } 12 . \\
\text { 18. } 12 . \\
\text { getötet }\end{array}$ & Negativ. \\
\hline 126 & $\begin{array}{l}1 \text { ccm klarer Urin, sehr } \\
\text { spärliche Leukocyten, } \\
\text { keine Tbc.-Bacillen. }\end{array}$ & 29.11. & $\begin{array}{l}\text { 10. } 12 . \\
\text { 18. } 12 . \\
\text { 27. } 12 .\end{array}$ & Negativ. \\
\hline
\end{tabular}

Aus der Tabelle geht hervor, dass die Tuberculose bei intralienaler Impfung im allgemeinen früher und ausgodehnter zur Entwicklung gelangt als bei intrahepatischer. Dies darf a priori nicht Wunder nehmen, da, wic Oppenheimer selbst hervorhebt, auch bei Leberimpfung oft die Erkrankung der $\mathrm{Hilz}$ noch ausgesprochener war 
als die der Leber, und, wie sich aus der Section vorzeitig getöteter Tiere ergab, früher in die Erscheinung trat". Von Interesse erscheint ferner, dass trotz der in manchen Fällen sehr fortgeschrittenen Erkrankung der Milz weder Jueber noch Drüsen, noch andere Organe tuberculöse Veränderungen aufwiesen. Besonders möchten wir hervorheben, dass die von Kiralyfi beobachtete Schwellung und Verkäsung der retrosternalen und retrojugularen Lymphdrüsen, die ein allererstes Zeichen der beginnenden allgemeinen Infection sein soll, bei unseren Obductionen sich nur dann fand, wenn schon stärkrre Veränderungen in der Milz nachweisbar waren. Anderseits fanden wir die Drüsen niemals verdächtig, wenn die Milz keine Lrkrankung aufwies.

Wir können daher die Befunde von Meyer und Jacoby, welche die Tuberculose fast ausnahmslos zuallererst in diesen Drüsen fanden, bevor noch irgend ein anderes Organ erkrankt war, nicht bestätigen. Allerdings haben diese $\Lambda$ utoren nur intraperitoneal inficiert.

Ueberhaupt ist es uns im Gegensatz zu diesen Autoren bei intraperitonealer Impfung niemals gelungen, nach 14 Tagen Tuberculose in rgend einem Organ, auch nicht in den Drüsen, mit Sicherheit nachizuweisen. Hin und wieder fanden sich zwar leicht geschwellte retrojugulare oder retrosternale Drüsen, die aber zu dieser Zeit noch keine Spur von Verkäsung zeigten und infolgedessen eine sichere Diagnose nicht zuliessen.

Zur richtigen Einschätzung des Wertes der Impfung in die Milz baben wir neben den Parallelversuchen mit Leberimpfung auch die Intraperitonealimpfung zum Vergleiche herangezogen. Wir konnten feststellen, dass zu Ende der zweiten Woche, zu welcher Zeit die meisten in die Milz geimpften Tiere ein positives Resultat ergaben, sich bei intraperitonealer Impfung noch keine Tuberculose nachweisen liess, was ja auch a priori vorauszusetzen war.

Nachdem wir uns überzeugt hatten, dass es mit Hilfe unserer Methode gelingt, in durchschnittlich zwei Wochen den Nachweis der Tuberculose zu erbringen, gingen wir daran, eine weitere Abkürzung des Nachweises durch Intracutanreaction zu versuchen. Doch sind wir damit zu kcinem Resultat gelangt

Eine Reihe von Versuchen lehrte uns, dass wir niemals eine typische Reaction auslösen konnten, bevor nicht eine makroskopisch nachweisbare Tuberculose des Versuchstieres bestand. Tatsächlich hat Römer die Reaction frühestens am 21. Tage angestellt und infolgedessen verlässliche Resultate erzielt. Die von uns in der zweiten Woche angestellten Reactionen waren entweder völlig ncgativ oder zeigten die von Römer und Joseph als atypisch bezeichnete Form, die nach den genannten Autoren nzwar nur nach der Infection mit Tuberculose auftritt, aber keine anatomische Tuberculose beweist". Mit dieser Reaction haben später Esch und Hagemann durchschnittlich nach 14 bis 18 Tagen, in oinzelnen Fällen schon nach 10 Tagen, hier und da allerdings erst nach drei Wochen positive Resultate erzielt. Beide Autoren geben allerdings zu, dass die Reaction erst positiv wird, wenn sich bei der Obduction des Versuchs- 
tieres 'l'uberculose nachweisen lïsst. Gerade dieses letztere Moment hat uns davon abgehalten, den Wert der Reaction an einem grossen Material durchzuprüfen. Immerhin haben wir in einigen Versuchen feststellen können, dass die Reaction in der zweiten Woche auch dann noch negativ ausfiel, wenn wir bei der Relaparotomie bereits Tuberculose feststellen konnten.

Was die Methode Hagemanns betrifft, dic den Nachweis der Tuberculose derart erbringt, dass an hochtuberculösen Tieren mit dem fraglichen Material eine Intracutanreaction gemacht wird, so haben wir uns mit derselben nicht beschäftigt. Die Methode, welehe ein positives Resultat bereits nach 48 Stunden ergeben soll, hat den Nachteil, dass man inmer hochtuberculöse Tiere vorrätig haben muss, die auf intracutane Alttuberculinimpfung ty pische Intracutanreactionen aufweisen müssen. $\Lambda$ us diesem Grunde ist sie nur in grossen Laboratorien ausführbar.

Wir haben schliesslich versucht, durch die von Jacoby und Heyer angegebene subcutane. Tuberculinreaction die Tuberculose des Versuchstieres möglichst frühzeitig zu ermitteln. $\Lambda$ ber auch mit dieser Methode konnten wir keinen Fortschritt orzielen. Die Reaction ist schon nach den Angaben von Esch und Hagemann weniger empfindlich, als die intracutane.

Im Anschluss an Versuche von Hagemann haben wir nur den Tod der Versuchsciere als positiv angesprochen, während wir aut Temperaturschwankungen nicht geachtet haben. Lus unseren 25 diesbezüglichen Versuchen geht eindeutig hervor, dass die Tubereulnse durch die subcutane Reaction erst bedeutend später nachweisbar ist, als dureh anatomischen Befund. Ja, es ist in drei Fällen sogar vorgekommen, dass bei anatomisch ziemlich ausgebreiteter Tuberculose auf eine subcutane Injection von $0,5 \mathrm{ccm}$ AT der Tod des Versuchstieres nicht erfolgte.

Diese Versuche lehrten uns, dass wir mit der intracutanen oder subcutanen Reaction nicht mehr erzielen konnten als durch Relaparotomie.

\section{Résumé.}

Nach dem Gesagton scheint uns die Impfung in parenchymatöse Organe die Methode der Wahl zum kürzesten biologischen Nachweise der Tuberculose zu sein. Zur Impfung eignet sich in erster Linie dic Milz des Meersehweinchens, in welcher nach durchschnittlich 14 T'agen Tuberculose makroskopisch nachweisbar ist. Als besonderen Vorteil unserer Methode betrachten wir auch die Relaparotomie, die es gestattet, ein Tier wiederholt aul das Vorhandensein von Tuberculose zu untersuchen.

Iss ist uns eine angenehme Pflicht, Herm Sanitätsrat Director Dr. Hofmokl für die Ueberlassung des Tiermaterials, sowie Herrn Vorstand Prof. Dr. Freund für vielfache freundliche Lnterstützung unseren besten Dank auszusprechen. 
288 Damask u. Sehweinburg, Nachw. d. Tuberculose inı Tiervers. d. Hilzimpfung.

\section{Literatur.}

1. Bacbrach und Necker, Wiener klin. Wochenschr. 1911. Nr. 12.

2. Blocb, Berliner klin. Wochenschr. 1907. Nr. 44.

3. Damsch, cit. nach Hagemann.

4. Dieterlen, T'uberculosearbeiten aus dem Kaiserl. Gesundheitsamte. 1908. II.9.

5. Esch, Münchener med. Wochenschr. 1912. Nr. 39.

6. Fraenkel, Berliner klin. Wochenschr. 1898. Nr. 40.

7. Hag e mann, R., Ueber die Diagnose chirurgischer'Tuberculose usw. Habilitationsschrift. Tübingen 1912, und Beitr. z. klin. Chir. Bd. 82. H. 1.

8. Dorselbe, Med. Klinik. 1913.

9. Jakoby, M. und Meyer, N., Beitr. z. Klinik der Tuborculose. 1911. Bd. 20.

10. Kiralyfi, Berliner klin. Wochenschr. 1910. Ni. 47.

11. Laabs, Inaug.-Diss. Freiburg 1894, cit. nach Rabinowitsch.

12. Oppenheimer, Mänchener med. Wochenschr. 1911. S. 2164 und 1912. S. 2817.

13. Pappenheim, Berliner klin. Wochenschr. 1898. Nr. 37.

14. Rabinowitsch, Deutsche med. Wochenschr. 1900. Nr. 16, und Berliner klin. Wochenschr. 1907. Nr. 2.

15. Römer, Beitr. z. Ḱlinik d. Tuberculose. 1909. Bd. 12. H. 1.

16. Römer und Joseph, Ebenda. 1909. Bd. 14.

17. Salus, Berliner klin. Wochenschr. 1906. S. 1150.

18. Zahn, Inaug.-Diss. Tübingen 1884, cit. nach Rabinowitsch. 